\title{
CXCL14 is a candidate biomarker for Hedgehog signalling in idiopathic pulmonary fibrosis
}

\author{
Guiquan Jia, ${ }^{1}$ Sanjay Chandriani, ${ }^{1}$ Alexander R Abbas, ${ }^{1}$ Daryle J DePianto, ${ }^{1}$ \\ Elsa N N'Diaye, ${ }^{1}$ Murat B Yaylaoglu, ${ }^{1}$ Heather M Moore, ${ }^{1}$ Ivan Peng, ${ }^{1}$ Jason DeVoss, ${ }^{1}$ \\ Harold R Collard, ${ }^{2}$ Paul J Wolters, ${ }^{2}$ Jackson G Egen, ${ }^{1}$ Joseph R Arron ${ }^{1}$
}

\begin{abstract}
- Additional material is published online only. To view please visit the journal online (http://dx.doi.org/10.1136/ thoraxjnl-2015-207682).

${ }^{1}$ Genentech, Inc., South San Francisco, California, USA ${ }^{2}$ Division of Pulmonary and Critical Care Medicine, Department of Medicine, University of California, San Francisco, California, USA
\end{abstract}

Correspondence to Dr Joseph R Arron, Genentech, Inc., MS 34, 1 DNA Way, South San Francisco, CA 94080, USA arron.joseph@gene.com

Received 9 August 2015 Revised 31 January 2017 Accepted 3 February 2017 Published Online First 1 March 2017

\begin{abstract}
Background Idiopathic pulmonary fibrosis (IPF) is associated with aberrant expression of developmental pathways, including Hedgehog (Hh). As Hh signalling contributes to multiple pro-fibrotic processes, Hh inhibition may represent a therapeutic option for IPF. However, no non-invasive biomarkers are available to monitor lung Hh activity.
\end{abstract}

Methods We assessed gene and protein expression in IPF and control lung biopsies, mouse lung, fibroblasts stimulated in vitro with sonic hedgehog $(\mathrm{SHh})$, and plasma in IPF patients versus controls, and cancer patients before and after treatment with vismodegib, a Hh inhibitor.

Results Lung tissue from IPF patients exhibited significantly greater expression of Hh-related genes versus controls. The gene most significantly upregulated in both IPF lung biopsies and fibroblasts stimulated in vitro with SHh was CXCL14, which encodes a soluble secreted chemokine whose expression is inhibited in vitro by the addition of vismodegib. CXCL14 expression was induced by SHh overexpression in mouse lung. Circulating CXCL14 protein levels were significantly higher in plasma from IPF patients than controls. In cancer patients, circulating CXCL14 levels were significantly reduced upon vismodegib treatment.

Conclusions CXCL14 is a systemic biomarker that could be used to identify IPF patients with increased Hh pathway activity and monitor the pharmacodynamic effects of Hh antagonist therapy in IPF.

Trial registration number Post-results, NCT00968981.

\section{INTRODUCTION}

Idiopathic pulmonary fibrosis (IPF) is a chronic, progressive and lethal fibrotic disease of unclear aetiology leading to irreversible scarring of the lung parenchyma and loss of lung function. ${ }^{1}$ Genetic predisposition to pulmonary fibrosis implicates type 2 alveolar epithelial cell (AEC2) dysfunction in disease pathogenesis and suggests that increased susceptibility of the alveolar epithelium to accumulated environmental insults promotes an aberrant wound healing response in the underlying mesenchymal cells, resulting in myofibroblast differentiation, proliferation and excessive extracellular matrix (ECM) deposition. ${ }^{2}$

Multiple molecular pathways are aberrantly expressed in IPF tissue including directly profibrotic mediators such as TGF $\beta$, IL13 and FGF. ${ }^{3}$ In addition, pathways implicated in embryonic

\section{Key messages}

What is the key question?

- How can activity of the profibrotic Hedgehog signalling pathway be monitored non-invasively in patients with idiopathic pulmonary fibrosis (IPF)?

\section{What is the bottom line?}

- Using human IPF lung tissue and blood, in vitro cell culture, in vivo animal models and blood samples from an interventional clinical study, we show that CXCL14 is a candidate blood biomarker of Hedgehog pathway activity in IPF.

\section{Why read on?}

- The ability to monitor the activity of key signalling pathways non-invasively may aid in the development of molecularly targeted therapies for IPF.

development and patterning such as Wnt and Hedgehog $(\mathrm{Hh})$ are reactivated in IPF and may contribute to fibrogenesis. ${ }^{4-10}$ Sonic Hedgehog (SHh) is essential for branching morphogenesis in the developing lung; ${ }^{11}$ overexpression of $\mathrm{SHh}$ in mouse embryos results in the accumulation of excessive mesenchymal tissue. ${ }^{12}$ In healthy adult tissue, there is minimal evidence for $\mathrm{Hh}$ activity. Bleomycin-mediated lung injury induces $\mathrm{Hh}$ pathway activity and SHh overexpression increases fibrotic collagen deposition in the context of bleomycin injury. ${ }^{13} \mathrm{Hh}$ activity in human lung fibroblasts can promote multiple profibrotic processes, including enhanced sensitivity to TGF $\beta$ and PDGF, leading to increased migration, contractility and survival, ${ }^{5}{ }^{6}$ while epithelial cell-derived $\mathrm{SHh}$ and fibroblast-derived TGF $\beta$ can reciprocally induce each other $;{ }^{14}$ these epithelial-mesenchymal interactions collectively can contribute to pathological fibrosis.

Canonical Hh signalling is a highly regulated process. In the constitutive unliganded 'off' state, patched transmembrane receptors (eg, PTCH1 and $\mathrm{PTCH} 2$ ) inhibit the activity of smoothened (SMO), maintaining its localisation to intracellular endosomes. When a secreted Hh ligand, for example, $\mathrm{SHh}$, binds to PTCH, SMO repression is released and SMO accumulates in the primary cilium, 
disrupting constitutive proteolysis of GLI transcription factors and resulting in translocation of intact GLI to the nucleus where it can activate transcription of target genes. Importantly, GLI itself is a transcriptional target of Hh pathway activity, resulting in a positive feedback loop. ${ }^{8} 15$

Basal cell carcinoma (BCC) of the skin is the most common form of cancer and is characterised by excessive activity of the Hh pathway. Individuals with Gorlin syndrome carry inactivating mutations in PTCH1, resulting in excessive $\mathrm{Hh}$ activity in the skin and multiple aggressive, recurrent BCCs. ${ }^{16}$ Vismodegib, a small molecule inhibitor of SMO, reduces the growth and invasiveness of locally advanced and metastatic BCC, and is an approved therapy for that indication. ${ }^{17}$

Targeting the $\mathrm{Hh}$ pathway may be a potential therapeutic option for IPF. However, molecular, pathological and clinical heterogeneity in IPF patients ${ }^{18}$ makes it difficult to monitor molecular responsiveness to treatment and identify those most likely to benefit from therapy without directly sampling lung tissue, which poses risks to patients and is logistically impractical in large multicentre clinical studies. Therefore, we sought to characterise Hh pathway activity in IPF lungs and identify candidate non-invasive biomarkers related to $\mathrm{Hh}$ activity that could be used as pharmacodynamic and/or predictive indicators of treatment effects.

\section{METHODS}

Detailed methods can be found in the online supplement.

\section{Patient samples}

All tissues, plasma samples and clinical data for IPF were obtained as described previously. ${ }^{19}$

SHH4610g was a multicentre, two-stage, open-label phase Ib study designed to describe the pharmacokinetics (PK) of vismodegib (GDC-0449, a small molecule SMO inhibitor) in 63 safety-evaluable patients with advanced solid tumours that were refractory to treatment or for whom no standard therapy existed. $^{20}$

\section{Cell culture and gene expression}

Human colon fibroblasts (CCD-18Co) were cultured per ATCC recommendations and treated in medium with recombinant human $\mathrm{SHh}$ protein and/or vismodegib. Gene expression was analysed using qPCR assays.

\section{Adenovirus mouse model}

C57BL/6J mice (Jackson Labs) were infected with recombinant, replication-deficient adenovirus (Vector Biolabs) encoding murine SHh (Ad-mSHh) or luciferase (Ad-Luc) by intratracheal (i.t.) administration or vehicle control. At 7 and 14 days after treatment, gene expression was analysed using qPCR assays. All animal experiments were approved by the Genentech Institutional Animal Care and Use Committee.

\section{Measurement of plasma protein biomarkers}

CXCL14 and CCL18 levels in EDTA-anticoagulated plasma were determined using CXCL14 and CCL18 DuoSet assays from R\&D Systems following manufacturer protocols with matrix optimisation for human plasma.

\section{Immunohistochemistry and in situ hybridisation}

Formalin-fixed paraffin-embedded lung tissue sections from IPF patients, obtained at the time of lung transplantation, and control lung tissue from unused donor lungs were examined by immunohistochemistry (IHC) for localisation of CXCL14 protein and non-isotopic RNA in situ hybridisation (ISH).

\section{Statistical analyses}

Statistical analyses were performed in R. Relationships between gene expression and plasma protein concentration across patient groups were tested by the Wilcoxon rank-sum method. Mouse lung gene expression was analysed by Student's t-test with Welch's correction for unequal SDs. Plasma biomarker changes over time were analysed by Student's paired t-test. Each timepoint was compared to baseline, with adjustment for multiple testing by the permutation method. A $\mathrm{p}$ value $<0.05$ was considered statistically significant. p Value correction for testing of multiple hypotheses in gene expression analyses was performed by applying the Benjamini-Hochberg method as implemented in the $\mathrm{R}$ package limma.

\section{RESULTS}

\section{Gene expression patterns in IPF implicate Hh pathway activity}

We have previously reported the assessment of gene expression in lung biopsies from patients with IPF $(\mathrm{N}=40)$ and control unused donor lung tissue $(\mathrm{N}=8)$ using $\mathrm{qPCR}^{19}$ and genomewide microarrays. ${ }^{21}$ In addition to evidence for activity of profibrotic signalling pathways such as ${\text { IL } 13^{19}}^{19}$ and consistent with other published reports, ${ }^{5} 6$ we observed transcriptional evidence for activity of the Hh signalling pathway. In the genome-wide analysis, Hh pathway transcription factors GLI1 (4.3-fold) and GLI2 (threefold) and the transmembrane activating protein SMO (2.6-fold) were among the significantly differentially upregulated transcripts in IPF as compared to control. To confirm the expression of these transcripts and explore other $\mathrm{Hh}$ pathway genes, we performed qPCR on the same biopsy samples. We observed slightly decreased expression of $\mathrm{SHH}$ and PTCH1 in IPF relative to control and significantly elevated expression of GLI1, GLI2, SMO and PTCH2 in IPF relative to control (figure 1). Taken together, these data suggest activity of the Hh signalling pathway in IPF lung tissue.

\section{CXCL14 is expressed at elevated levels in IPF lungs and is} inducible by SHh in vitro and in vivo

Previous studies of vismodegib assessed GLI1 expression in skin biopsies and hair follicles as a pharmacodynamic biomarker of $\mathrm{Hh}$ pathway activity in the skin. ${ }^{22}$ As GLI1 is a transcription factor primarily expressed in mesenchymal cells in lung tissue, its expression would be difficult to assess non-invasively in IPF patients. To identify candidate biomarkers of $\mathrm{Hh}$ activity in IPF lung, we looked for genes encoding soluble secreted proteins by comparing transcriptome-wide expression patterns of genes encoding secreted proteins in CCD-18Co human colon myofibroblast cells stimulated in vitro with $\mathrm{SHh}^{23}$ and IPF versus control lungs. ${ }^{21}$ The most significantly differentially expressed gene common to both datasets was CXCL14, which encodes a soluble chemokine. CXCL14 had a mean expression level 16.8-fold higher in IPF than in control lung tissue and 7.3-fold higher in SHh stimulated versus control CCD-18Co cells. Other genes encoding secreted proteins that were differentially expressed in both datasets included VCAN, IGFB7 and SLIT1; however, VCAN and IGFB7 were only modestly upregulated to an average of twofold in IPF versus control, and SLIT1 was downregulated in IPF (table 1). We confirmed differential CXCL14 expression by qPCR in IPF lung tissue (figure 2A) and in $\mathrm{CCD}-18 \mathrm{Co}$ cells upon $\mathrm{SHh}$ stimulation (figure 2B). SHh-mediated CXCL14 induction in CCD-18Co cells was 

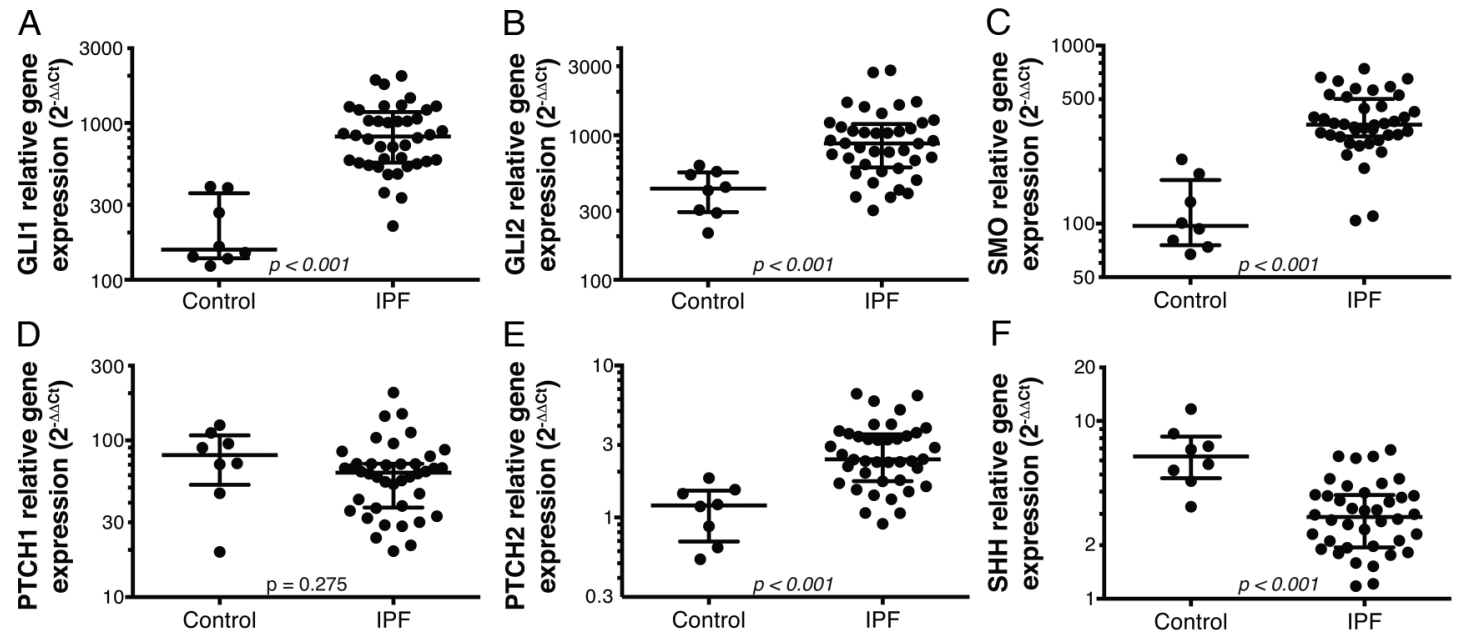

Figure 1 Expression levels of hedgehog ( $\mathrm{Hh}$ ) pathway genes in idiopathic pulmonary fibrosis (IPF) and control lung biopsy tissue. Expression of GLI1 (A), GLI2 (B), smoothened (SMO) (C), PTCH1 (D), PTCH2 (E) and sonic hedgehog (SHH) (F) genes were assessed by qPCR in bulk lung biopsy tissue from IPF patients $(\mathrm{N}=40)$ and control unused donor lung tissue $(\mathrm{N}=8)$. Wide lines denote median values and whiskers denote IQRs. $\mathrm{p}$ Values are Wilcoxon rank-sum.

Table 1 Significantly upregulated genes encoding secreted proteins upon SHh stimulation in CCD-18Co cells

\begin{tabular}{|c|c|c|c|c|c|c|}
\hline $\begin{array}{l}\text { Affymetrix } \\
\text { probe ID }\end{array}$ & $\begin{array}{l}\text { Gene } \\
\text { symbol }\end{array}$ & Gene name & $\begin{array}{l}\log _{2} \text { FC SHh vs } \\
\text { control }\end{array}$ & $\begin{array}{l}\text { Adjusted p } \\
\text { value }\end{array}$ & $\begin{array}{l}\log _{2} \text { FC IPF vs } \\
\text { control }\end{array}$ & $\begin{array}{l}\text { Adjusted } \\
\mathrm{p} \text { value }\end{array}$ \\
\hline 218002_s_at & CXCL14 & chemokine (C-X-C motif) ligand 14 & 2.8 & $<0.001$ & 4.1 & $<0.001$ \\
\hline 215646_s_at & VCAN & versican & 2.1 & $<0.001$ & 1.0 & $<0.001$ \\
\hline 213652_at & PCSK5 & proprotein convertase subtilisin/kexin type 5 & 1.5 & $<0.001$ & 0.33 & 0.089 \\
\hline 211312_s_at & WISP1 & WNT1 inducible signalling pathway protein 1 & 1.5 & 0.001 & 0.30 & 0.499 \\
\hline 210513_s_at & VEGFA & vascular endothelial growth factor A & 1.4 & $<0.001$ & -0.72 & 0.032 \\
\hline 222719_s_at & PDGFC & platelet derived growth factor $C$ & 1.3 & $<0.001$ & 0.33 & 0.089 \\
\hline 210683_at & NRTN & neurturin & 1.3 & $<0.001$ & -0.46 & 0.002 \\
\hline 210150_s_at & LAMA5 & laminin, $\alpha 5$ & 1.3 & $<0.001$ & -0.57 & $<0.001$ \\
\hline 230130_at & SLIT2 & slit homolog 2 (Drosophila) & 1.2 & $<0.001$ & -1.2 & $<0.001$ \\
\hline 213910_at & IGFBP7 & insulin-like growth factor binding protein 7 & 1.1 & $<0.001$ & 1.0 & $<0.001$ \\
\hline 213131_at & OLFM1 & olfactomedin 1 & 1.1 & $<0.001$ & 0.19 & 0.382 \\
\hline
\end{tabular}

inhibited by vismodegib treatment (figure 2C). Taken together, these data show that CXCL14 expression is elevated in IPF lungs; CXCL14 is a Hh-inducible gene whose expression can be inhibited by vismodegib in fibroblasts.

\section{CXCL14 expression is induced by lung overexpression of $\mathrm{SHh}$ in vivo}

To determine whether Hh activity can regulate CXCL14 expression in vivo, we monitored gene expression in the lungs of wildtype mice following intratracheal infection with a replicationdeficient adenoviral vector engineered to express murine $\mathrm{SHh}$ (Ad-mSHh). Controls for these studies included adenovirus expressing luciferase (Ad-Luc) to account for non-specific effects of infection and vehicle alone without virus (PBS). At 7 and 14 days after adenovirus infection, mice infected with Ad-mSHh expressed significantly higher levels of $\mathrm{SHH}$, GLI1, PTCH1, PTCH2 and CXCL14 compared to those infected with Ad-Luc or treated with PBS (figure 3). Increased SHH expression demonstrates sufficient viral infection and sustained Hh ligand expression; increases in GLI1, PTCH1 and PTCH2 show that the adenovirus-mediated $S H H$ expression leads to $\mathrm{Hh}$ pathway activation and target gene transcription; and induction of CXCL14 shows that CXCL14 is a transcriptional target of $\mathrm{SHh}$ activity in vivo in lung tissue.

\section{Localisation of CXCL14 protein and gene expression to} fibrotic regions of IPF lung

Lung tissue from IPF patients exhibits substantial spatial heterogeneity, with areas of normal-appearing lung parenchyma interspersed with myofibroblastic foci and dense scar tissue containing honeycomb change typical of a usual interstitial pneumonia (UIP) pattern. ${ }^{21} 24$ To characterise the spatial distribution of CXCL14 protein and CXCL14 gene expression, respectively, in IPF, we performed IHC and ISH on explanted lung tissue from IPF patients $(\mathrm{N}=6)$ and control unused donor lung tissue $(\mathrm{N}=3)$. CXCL14 protein immunoreactivity was dramatically increased in IPF compared to control, with intense staining in areas of AEC2 hyperplasia and bronchiolisation, and more diffuse staining in fibroblastic 


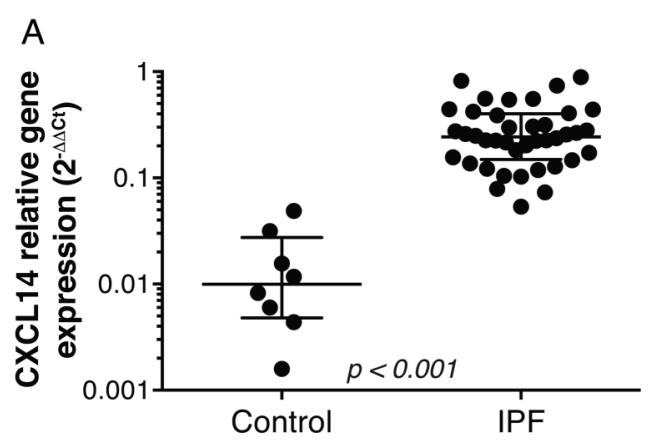

B
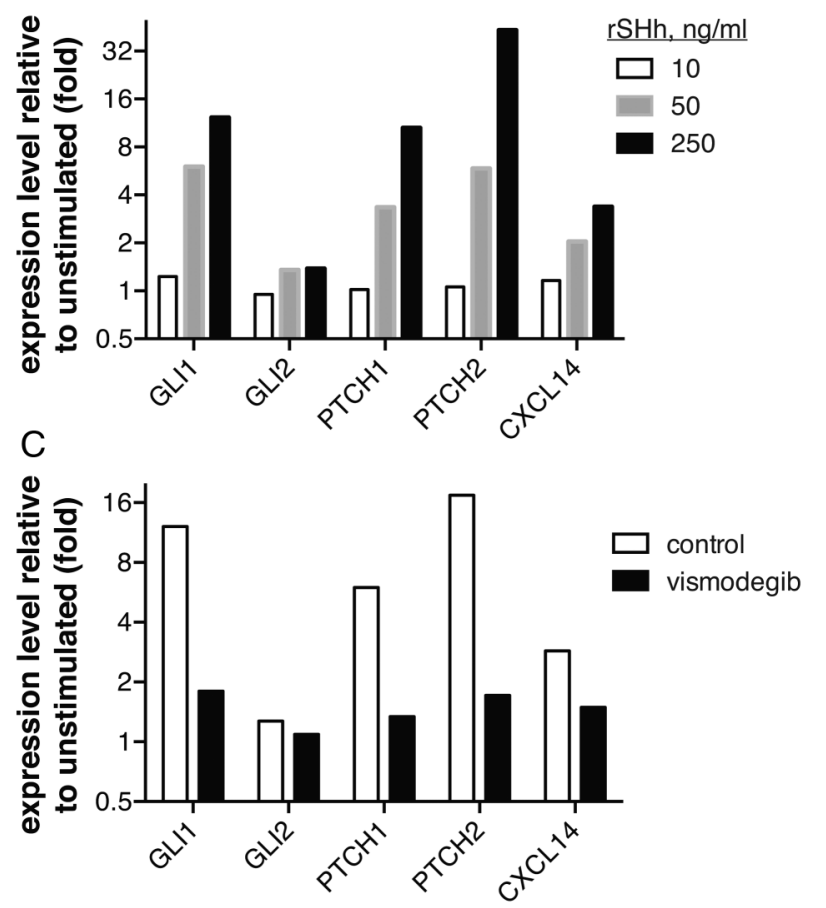

Figure 2 CXCL14 gene expression is elevated in idiopathic pulmonary fibrosis (IPF) lung biopsies, and is inducible in vitro by sonic hedgehog (SHh) and inhibited by vismodegib treatment in fibroblasts. (A) CXCL14 expression by qPCR as in figure 1. (B) CCD-18Co human fibroblasts were treated for 24 hours with the indicated doses of recombinant SHh (rSHh) and gene expression was assessed by qPCR. (C) As in (B), cells were treated with $250 \mathrm{ng} / \mathrm{mL} \mathrm{rSHh}$ with or without $100 \mathrm{nM}$ vismodegib or DMSO (vehicle control).

areas underlying relatively intact-appearing parenchymal tissue (figure 4A-D). CXCL14 gene expression was primarily localised in epithelial cells and underlying fibroblasts in areas of dense fibrosis in IPF lung tissue but was virtually undetectable in control lung (figure $4 \mathrm{E}-\mathrm{H}$ ). Control staining for IHC and ISH is shown in online supplementary figures S1 and S2. Taken together, these data show that CXCL14 gene and protein expression are increased in fibrotic regions of IPF lung tissue.

CXCL14 protein levels are elevated in plasma from IPF and cancer patients; vismodegib has pharmacodynamic effects on plasma CXCL14 levels in cancer patients

To explore the potential of CXCL14 protein as a systemic biomarker, we measured CXCL14 levels in plasma from IPF $(\mathrm{N}=80)$ and control $(\mathrm{N}=10)$ subjects using an immunoassay. Like many chemokines, CXCL14 interacts with heparin, ${ }^{25}$ which could lead to assay interference in serum or heparinised plasma, and so we optimised the CXCL14 assay for
EDTA-anticoagulated plasma. Plasma CXCL14 levels were significantly higher in IPF patients relative to control subjects, with approximately $70 \%$ of IPF patients exhibiting levels greater than the upper limit observed in a set of healthy controls assessed simultaneously (figure 5A). We next sought to determine whether Hh pathway inhibition with vismodegib exerted pharmacodynamic effects on circulating CXCL14 levels. As clinical samples from vismodegib-treated IPF patients were not currently available, we used plasma samples collected from solid tumour patients enrolled in a phase 1B dose-scheduling study of vismodegib. ${ }^{20}$ At baseline, the cancer patients in the vismodegib study $(\mathrm{N}=51)$ had elevated plasma CXCL14 levels relative to a separate cohort of healthy controls $(\mathrm{N}=18)$, exhibiting a similar distribution to that observed in IPF patients (figure 5B). Prior analyses suggested that weekly dosing with vismodegib in that study after an 11-dose daily run-in period did not provide adequate systemic coverage to mediate biological effects, and so we restricted pharmacodynamic analyses to patients in the daily (QD) or three times weekly (TIW) arms of the study. ${ }^{20}$ As approximately two thirds of cancer patients exhibited baseline elevation of plasma CXCL14 levels above the upper limit observed in a different set of control subjects assessed simultaneously $(0.85 \mathrm{ng} / \mathrm{mL})$, we hypothesised that any pharmacodynamic effects of vismodegib treatment might only be detectable in those patients. Consistent with that hypothesis, in patients with elevated baseline levels of plasma CXCL14 (greater than $0.85 \mathrm{ng} / \mathrm{mL}, \mathrm{N}=15$ ), there was a significant decrease in plasma CXCL14 levels upon vismodegib treatment, with median decreases of $22 \%$ and $48 \%$ after 28 and 49 days of treatment, respectively (figure $5 \mathrm{C}$ ). Four days after cessation of treatment, there appeared to be a slight rebound in plasma CXCL14 levels, with a median decrease of $15 \%$ compared to baseline at day 57 . We did not observe any significant pharmacodynamic effect of vismodegib treatment on patients with baseline CXCL14 levels in the 'normal' range (less than $0.85 \mathrm{ng} / \mathrm{mL}, \mathrm{N}=8$; figure $5 \mathrm{D}$ ). To assess whether this change over time reflected reversion to the mean or other unrelated effects, we assessed plasma levels of CCL18, a chemokine not known to be regulated by Hh signalling, and did not observe any pharmacodynamic effects of vismodegib treatment on plasma CCL18 levels despite systemically elevated levels in cancer patients in the study relative to controls (see online supplementary figure S3). Taken together, these data show that blood levels of CXCL14 protein are significantly elevated in IPF patients compared to controls, and in solid tumour patients with elevated baseline levels of CXCL14, there was a specific and significant decrease in systemic CXCL14 upon $\mathrm{Hh}$ pathway inhibition with vismodegib treatment.

\section{DISCUSSION}

Although Hh signalling is critical for embryonic lung development, the pathway is largely dormant in healthy adult lung tissue. $^{2} 1318$ Reactivation of the $\mathrm{Hh}$ signalling pathway is a feature of fibrotic lung tissue in IPF. Hh signalling can contribute to fibrogenesis, including cross-talk with other pathways active in IPF such as TGF $\beta$, FGF, PDGF and IL13, resulting in increased myofibroblast differentiation, ECM production, motility and survival. . $^{-6} \quad 9 \quad 14 \quad 19 \quad 26 \quad 27$ Therefore, pharmacologic modulation of the Hh pathway may offer therapeutic benefit to patients with IPF. However, there is uncertainty as to whether Hh pathway activity in IPF is 'canonical' and subject to $\mathrm{Hh}$ pathway inhibitors such as vismodegib, and IPF pathogenesis is heterogeneous across patients. Lacking an established means to assess the activity of the Hh pathway in lung tissue non- 

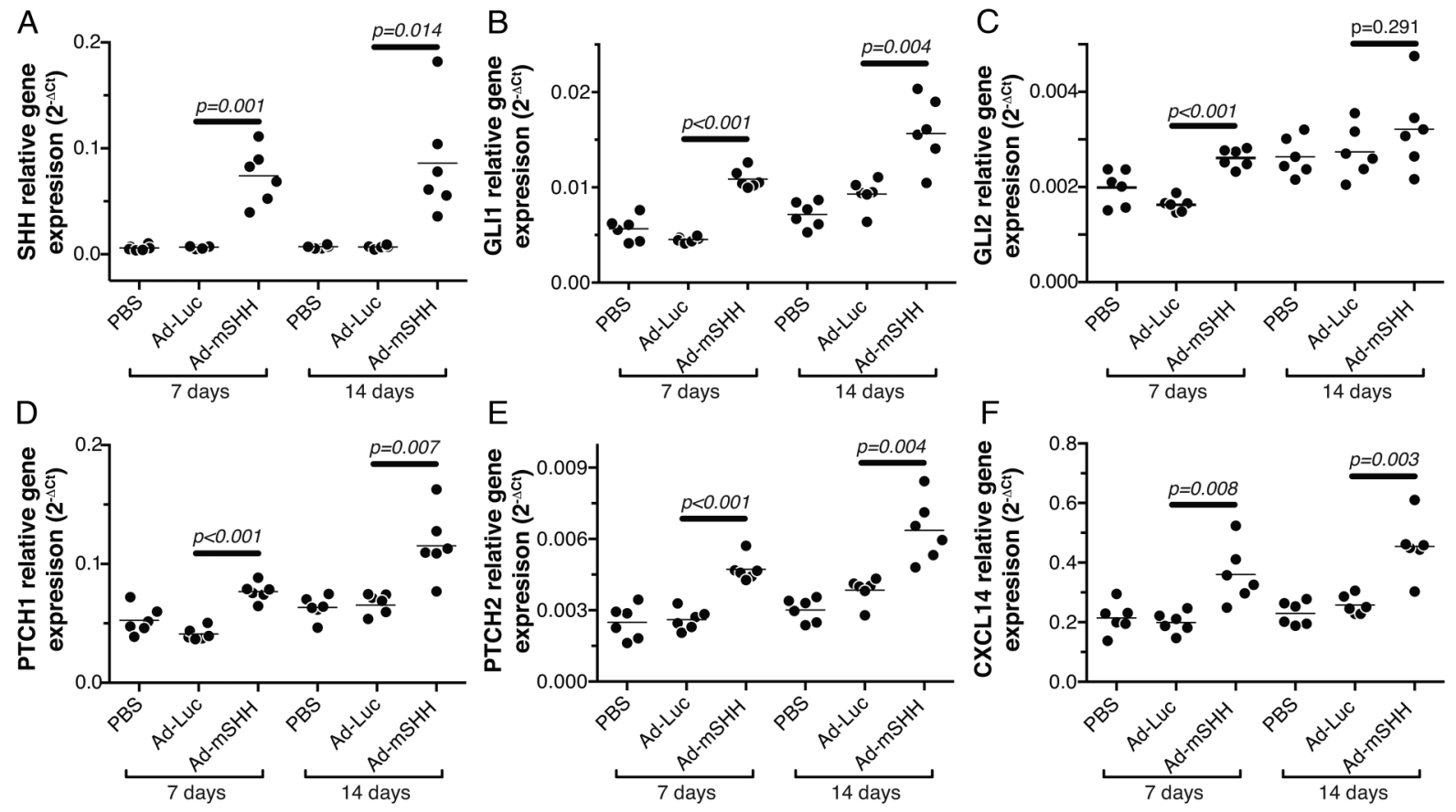

invasively, we sought to identify a peripheral biomarker reflecting $\mathrm{Hh}$ activity that might have clinical utility in IPF. Here, we show through a complementary series of in vitro and in vivo experiments that: (1) CXCL14 gene and protein expression levels are dramatically elevated in IPF lung tissue; (2) CXCL14 is the most highly upregulated gene encoding a secreted protein in SHh-treated fibroblasts: (3) ectopic expression of $\mathrm{SHh}$ in mouse lung induces CXCL14 expression; (4) peripheral blood concentrations of CXCL14 protein are significantly elevated in both IPF and solid tumour patients; and (5) vismodegib treatment results in pharmacodynamic modulation of CXCL14 levels in cancer patients. Therefore, we propose CXCL14 as a candidate blood biomarker for Hh in IPF.

As IPF is a complex and heterogeneous disease with challenging clinical outcomes to assess, biomarkers may help with the design and interpretation of interventional studies of therapeutic candidates. ${ }^{28}$ CXCL14 may be used as a pharmacodynamic biomarker to assess the extent of Hh pathway inhibition mediated by SMO inhibitors. It may also serve as a predictive biomarker that identifies IPF patients with high Hh pathway activity and hence most likely to exhibit a clinical response to treatment. Although lung CXCL14 expression is universally elevated and CXCL14 plasma levels are continuously distributed in IPF patients, there are precedents for enriching for benefit in clinical studies of molecularly targeted therapies in various indications by selecting patients using median levels of continuously distributed biomarkers. ${ }^{29}$ If CXCL14 proves to be a specific biomarker of Hh pathway activity in IPF, changes in CXCL14 levels upon inhibition of other molecular pathways known to be active in IPF (eg, TGF $\beta$, IL13, PDGF, FGF, etc) may indicate the degree of cross-talk between those pathways and Hh signalling.

While other studies have reported conflicting findings with respect to the expression levels of some of the components of the Hh signalling pathway in IPF biopsies, ${ }^{4-6} 9$ elevated expression and/or nuclear localisation of GLI1 and GLI2 are generally held to be the defining characteristics of Hh pathway activity in vivo and are consistent across the published studies and our findings. The differential expression of $S H H$ and SMO may reflect differences in cellularity in the bulk biopsy samples in IPF rather than true transcriptional regulation due to signalling as IPF samples are likely to have increased ratios of fibroblasts to epithelial cells.

There is some question as to whether targeting SMO will inhibit the Hh pathway activity observed in IPF. In vitro data from lung fibroblasts have suggested that TGF $\beta$ can induce GLI1 activity independently of Hh ligand and SMO signalling. ${ }^{6}$ Preclinical animal models have shown that GLI-expressing mesenchymal cells are increased during bleomycin-induced lung fibrosis and that GANT61, a small molecule that inhibits GLI transcriptional activity downstream of SMO, has greater antifibrotic activity than vismodegib, which operates at the level of SMO. ${ }^{30}$ Although vismodegib dosing may have been suboptimal in that study $(40 \mathrm{mg} / \mathrm{kg}$ intraperitoneally every other day as compared to daily oral treatment in tumour xenograft models showing saturation at doses at or above $50 \mathrm{mg} / \mathrm{kg}$ per $\mathrm{os}^{31}$ ), another report claimed that therapeutic administration of $\mathrm{Hh}$ ligand blocking antibodies did not ameliorate bleomycininduced lung fibrosis, ${ }^{13}$ which suggests that Hh ligand-induced signalling may not be required for fibrosis in the bleomycin model. However, the relevance of the bleomycin injury model to human IPF has shown limited translatability, ${ }^{32}$ and the expression levels of $\mathrm{Hh}$ pathway components reported in those studies do not mirror what has been observed in human IPF. Importantly, adenovirus-mediated overexpression of $\mathrm{SHh}$ in combination with bleomycin treatment led to increased collagen deposition in the lungs of mice, indicating that Hh ligands can 
Figure 4 Localisation of CXCL14 protein and gene expression in idiopathic pulmonary fibrosis (IPF) lung tissue. Immunohistochemistry (A-D) or in situ hybridisation $(\mathrm{E}-\mathrm{H})$ was performed for CXCL14 protein and mRNA expression, respectively. Representative images shown from observation of six separate IPF and three control biopsies, as indicated. Images on right represent boxed regions from images on left. In panels $A$ and $E$, arrowheads indicate pleural surfaces; ' $p$ ' denotes normal-appearing parenchyma, ' $f$ ' denotes regions of dense fibrosis, and ' $b$ ' denotes bronchiolization.
Figure 5 Plasma CXCL14 protein levels are elevated in idiopathic pulmonary fibrosis (IPF) and cancer patients, and decrease in response to vismodegib treatment. (A) Plasma CXCL14 levels in healthy control $(\mathrm{N}=10)$ and IPF patients $(\mathrm{N}=80)$. (B) Plasma CXCL14 levels in a separate group of healthy control $(\mathrm{N}=18)$ and cancer patients enrolled in the SHH4610g study at baseline $(\mathrm{N}=51)$. (C) Plasma CXCL14 levels in

'CXCL14-high' cancer patients $(\mathrm{N}=15)$ over time after vismodegib treatment; patients with baseline levels $>0.85 \mathrm{ng} / \mathrm{mL}$ shown. (D) As in (C) for 'CXCL14-low' cancer patients (baseline levels $<0.85 \mathrm{ng} / \mathrm{mL} ; \mathrm{N}=8$ ). ( $($ and $B$ ) Wide lines denote median values and whiskers denote IQRs; $p$ values are Wilcoxon rank-sum. (C and D) Dashed lines connect matched longitudinal samples from individual subjects; $p$ values are versus baseline levels at each timepoint by Student's t-test with multiple testing adjustment by permutation.
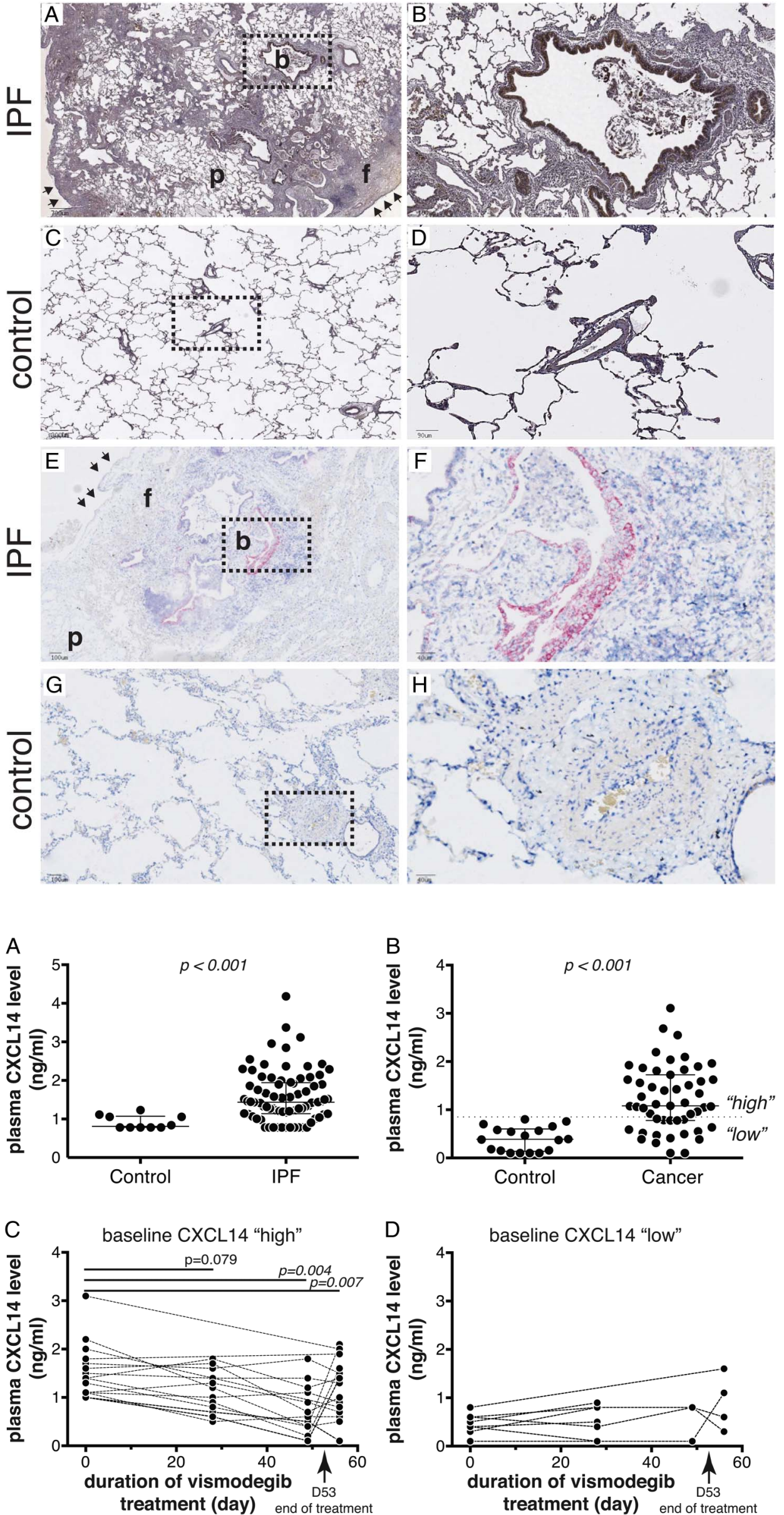
exacerbate fibrotic responses. ${ }^{13}$ The uncertainty associated with preclinical IPF models emphasises that in interventional clinical studies of SMO antagonists such as vismodegib in human IPF, it will be important to determine whether the intervention inhibits the Hh pathway activity observed in IPF lungs to assess clinical efficacy (or lack thereof). A non-invasive biomarker of $\mathrm{Hh}$ pathway activity in IPF will therefore be of great value to interpret outcome data.

CXCL14 (also known as BRAK) is a chemokine of the C-X-C family. Although its in vivo cellular receptor(s) have yet to be definitively identified, CXCL14 has been reported to exhibit chemotactic activity towards various inflammatory mononuclear cells ${ }^{33-36}$ or to act as a natural inhibitor of CXCL12-CXCR4 interactions. ${ }^{37} 38$ CXCL14-deficient mice are viable and fertile with no obvious immune defects, ${ }^{39}$ however they appear to be protected in obesity-induced insulin resistance models. ${ }^{40}{ }^{41} \mathrm{In}$ preclinical hepatic fibrosis models, CXCL14 expression is elevated in response to carbon tetrachloride-induced liver injury, ${ }^{42}{ }^{43}$ bile duct ligation ${ }^{43}$ and schistosome infection; ${ }^{44}$ multiple hepatic fibrosis models are $\mathrm{Hh}$ dependent. ${ }^{45}$ Elevated CXCL14 expression has been reported in association with multiple tumour types, and it has been reported as an autocrine growth factor for fibroblasts and as being involved in prostate tumour growth. ${ }^{46}{ }^{47}$ In an experimental system analogous to the one we used here, CXCL14 was found to be highly induced by $\mathrm{SHh}$ in a prostatic mesenchymal cell line and its expression co-localised with SHh pathway components in embryonic prostate tissue. ${ }^{48}$ In lung epithelium, CXCL14 expression has been reported to be slightly elevated in healthy smokers, with substantially greater expression in patients with COPD, and lung adenocarcinomas. High CXCL14 expression in tumour samples was associated with decreased survival in lung cancer patients. ${ }^{49}$ CXCL14 levels are increased in pancreatic cancer tissues and are associated with tumour invasiveness; ${ }^{50}$ given the strong association of $\mathrm{Hh}$ pathway activity with pancreatic cancer, ${ }^{51}$ it will be of interest to determine whether CXCL14 expression is regulated by Hh signalling in this context.

Our study has several limitations. We cannot definitively conclude that the excess plasma CXCL14 levels in IPF patients are derived from lung tissue. However, given that solid tumour patients exhibited elevated plasma CXCL14 levels and systemic Hh pathway antagonism with vismodegib significantly reduced circulating CXCL14 levels, we infer that the disease process in the lungs of IPF patients contributes sufficient excess CXCL14 to the systemic pool to account for the overall increase in plasma CXCL14 in IPF. While the Hh pathway activity in solid tumours is susceptible to SMO antagonism, it remains to be determined whether the same is true of IPF, thus the pharmacodynamic effect of vismodegib on CXCL14 in cancer may not translate to IPF. The cellular provenance of CXCL14 is unclear: while Hh ligands are conventionally thought to derive from epithelial cells and act on mesenchymal cells, CXCL14 protein and gene expression appear to localise to epithelial regions (figure 4). However, there is evidence for nuclear GLI1 and GLI2 localisation in both epithelium and fibroblasts in $\mathrm{IPF}^{5}$ while other studies have shown Hh pathway activation in both epithelium and mesenchymal cells in lung adenocarcinoma, ${ }^{52}$ BCC $^{53}$ and taste bud papillae, ${ }^{54}$ all dependent on epithelial-mesenchymal interactions.

In conclusion, reactivation of the developmental Hh pathway is hypothesised to be a pathogenic mechanism in IPF. We have shown evidence for elevated activity of the Hh pathway in human IPF lung tissue. Using a series of complementary in vitro and in vivo experiments, we have identified the chemokine CXCL14 as a candidate peripheral biomarker of Hh pathway activity that may have clinical utility in interventional studies of Hh pathway modulators and other candidate therapeutics in IPF.

Acknowledgements We thank the patients who participated in these studies and the providers who referred patients to the UCSF ILD clinic. Without the generous donation of biological samples from patients, this study could not have been performed. We also thank Rick Graham, Bob Yauch, Peter Dijkgraaf and Fred de Sauvage (Genentech) for scientific and technical advice on Hh signalling and vismodegib experiments.

Contributors GJ, SC, ARA, DJDeP, ENN, MBY, HMM, IP and JDeV designed, executed and analysed experiments. JGE and JRA conceived of and directed the studies. HRC and PJW enrolled IPF patients and collected samples. GJ, JGE and JRA wrote the manuscript. All authors reviewed and approved the content of the manuscript.

Funding Genentech, the Nina Ireland Program for Lung Health at UCSF, and NIH grant HL 108794.

Competing interests GJ, SC, ARA, DJDeP, ENN, MBY, HMM, IP, JDeV, JGE and JRA are or were employees of Genentech, Inc. when this work was conducted and may hold stock or stock options in the Roche Group. GJ, SC, ARA, DJDeP, JGE and JRA are named inventors on patents pending related to the diagnosis and treatment of pulmonary fibrosis.

Ethics approval UCSF Committee on Human Research

Provenance and peer review Not commissioned; externally peer reviewed.

\section{REFERENCES}

1 Adkins JM, Collard HR. Idiopathic pulmonary fibrosis. Semin Respir Crit Care Med 2012;33:433-9.

2 Leslie KO. Idiopathic pulmonary fibrosis may be a disease of recurrent, tractional injury to the periphery of the aging lung: a unifying hypothesis regarding etiology and pathogenesis. Arch Pathol Lab Med 2012;136:591-600.

3 Wynn TA. Integrating mechanisms of pulmonary fibrosis. J Exp Med 2011;208:1339-50.

4 Stewart GA, Hoyne GF, Ahmad SA, et al. Expression of the developmental Sonic hedgehog (Shh) signalling pathway is up-regulated in chronic lung fibrosis and the Shh receptor patched 1 is present in circulating T lymphocytes. I Pathol 2003:199:488-95.

5 Bolaños AL, Milla CM, Lira JC, et al. Role of Sonic Hedgehog in idiopathic pulmonary fibrosis. Am J Physiol Lung Cell Mol Physiol 2012;303:L978-90.

6 Cigna N, Farrokhi Moshai E, Brayer $S$, et al. The hedgehog system machinery controls transforming growth factor- $\beta$-dependent myofibroblastic differentiation in humans: involvement in idiopathic pulmonary fibrosis. Am J Pathol 2012;181:2126-37.

7 Chilosi M, Poletti V, Zamò A, et al. Aberrant Wnt/beta-catenin pathway activation in idiopathic pulmonary fibrosis. Am J Pathol 2003;162:1495-502.

8 Konigshoff M, Balsara N, Pfaff EM, et al. Functional Wnt signaling is increased in idiopathic pulmonary fibrosis. PLOS ONE 2008;3:e2142.

9 Coon DR, Roberts DJ, Loscertales M, et al. Differential epithelial expression of SHH and FOXF1 in usual and nonspecific interstitial pneumonia. Exp Mol Pathol 2006:80:119-23.

10 Kugler $\mathrm{MC}$, Joyner $\mathrm{AL}$, Loomis $\mathrm{CA}$, et al. Sonic hedgehog signaling in the lung. from development to disease. Am J Respir Cell Mol Biol 2015;52:1-13.

11 Pepicelli CV, Lewis PM, McMahon AP. Sonic hedgehog regulates branching morphogenesis in the mammalian lung. Curr Biol 1998;8:1083-6.

12 Bellusci S, Furuta Y, Rush MG, et al. Involvement of Sonic hedgehog (Shh) in mouse embryonic lung growth and morphogenesis. Development 1997;124:53-63.

13 Liu L, Kugler MC, Loomis CA, et al. Hedgehog signaling in neonatal and adult lung. Am J Respir Cell Mol Biol 2013;48:703-10.

$14 \mathrm{Hu} \mathrm{B}$, Liu J, Wu Z, et al. Reemergence of hedgehog mediates epithelial-mesenchymal crosstalk in pulmonary fibrosis. Am J Respir Cell Mol Biol 2015:52:418-28.

15 Rubin LL, de Sauvage FJ. Targeting the Hedgehog pathway in cancer. Nat Rev Drug Discov 2006:5:1026-33.

16 Mohan SV, Chang AL. Advanced basal cell carcinoma: epidemiology and therapeutic innovations. Curr Dermatol Rep 2014;3:40-5.

17 Sobanko JF, Okman J, Miller C. Vismodegib: a hedgehog pathway inhibitor for locally advanced and metastatic basal cell carcinomas. J Drugs Dermatol 2013;12 s154-5.

18 Travis WD, Costabel U, Hansell DM, et al. An official American Thoracic Society/ European Respiratory Society statement: update of the international multidisciplinary 
classification of the idiopathic interstitial pneumonias. Am J Respir Crit Care Med 2013;188:733-48.

19 Chandriani S, DePianto DJ, N'Diaye EN, et al. Endogenously expressed IL-13R $\alpha 2$ attenuates IL-13-mediated responses but does not activate signaling in human lung fibroblasts. J Immunol 2014;193:111-19.

20 Lorusso PM, Jimeno A, Dy G, et al. Pharmacokinetic dose-scheduling study of hedgehog pathway inhibitor vismodegib (GDC-0449) in patients with locally advanced or metastatic solid tumors. Clin Cancer Res 2011;17:5774-82.

21 DePianto DJ, Chandriani S, Abbas AR, et al. Heterogeneous gene expression signatures correspond to distinct lung pathologies and biomarkers of disease severity in idiopathic pulmonary fibrosis. Thorax 2015;70:48-56.

22 LoRusso PM, Rudin CM, Reddy JC, et al. Phase I trial of hedgehog pathway inhibitor vismodegib (GDC-0449) in patients with refractory, locally advanced or metastatic solid tumors. Clin Cancer Res 2011;17:2502-11.

23 Chen W, Tang T, Eastham-Anderson J, et al. Canonical hedgehog signaling augments tumor angiogenesis by induction of VEGF-A in stromal perivascular cells. Proc Natl Acad Sci USA 2011:108:9589-94.

24 Cavazza A, Rossi G, Carbonelli C, et al. The role of histology in idiopathic pulmonary fibrosis: an update. Respir Med 2010;104(Suppl 1):S11-22.

25 Tanegashima K, Suzuki K, Nakayama Y, et al. Antibody-assisted enhancement of biological activities of CXCL14 in human monocytic leukemia-derived THP-1 cells and high fat diet-induced obese mice. Exp Cell Res 2010;316:1263-70.

26 Syn WK, Witek RP, Curbishley SM, et al. Role for hedgehog pathway in regulating growth and function of invariant NKT cells. Eur J Immunol 2009;39:1879-92.

27 Furmanski AL, Saldana Jl, Ono M, et al. Tissue-derived hedgehog proteins modulate Th differentiation and disease. J Immunol 2013:190:2641-9.

28 Townsend MJ, Arron JR. Reducing the risk of failure: biomarker-guided trial design. Nat Rev Drug Discov 2016:15:517-18.

29 Arron JR, Townsend MJ, Keir ME, et al. Stratified medicine in inflammatory disorders: from theory to practice. Clin Immunol 2015;161:11-22.

30 Moshai EF, Wémeau-Stervinou L, Cigna N, et al. Targeting the hedgehogglioma-associated oncogene homolog pathway inhibits bleomycin-induced lung fibrosis in mice. Am J Respir Cell Mol Biol 2014;51:11-25.

31 Wong $H$, Alicke B, West KA, et al. Pharmacokinetic-pharmacodynamic analysis of vismodegib in preclinical models of mutational and ligand-dependent Hedgehog pathway activation. Clin Cancer Res 2011;17:4682-92.

32 Moeller A, Ask K, Warburton D, et al. The bleomycin animal model: a useful tool to investigate treatment options for idiopathic pulmonary fibrosis? Int I Biochem Cell Biol 2008:40:362-82.

33 Shellenberger TD, Wang M, Gujrati M, et al. BRAK/CXCL14 is a potent inhibitor of angiogenesis and a chemotactic factor for immature dendritic cells. Cancer Res 2004:64:8262-70.

34 Shurin GV, Ferris RL, Tourkova IL, et al. Loss of new chemokine CXCL14 in tumor tissue is associated with low infiltration by dendritic cells (DC), while restoration of human CXCL14 expression in tumor cells causes attraction of DC both in vitro and in vivo. J Immunol 2005;174:5490-8

35 Starnes T, Rasila KK, Robertson MJ, et al. The chemokine CXCL14 (BRAK) stimulates activated NK cell migration: implications for the downregulation of CXCL14 in malignancy. Exp Hematol 2006;34:1101-5.
36 Salogni L, Musso T, Bosisio D, et al. Activin A induces dendritic cell migration through the polarized release of CXC chemokine ligands 12 and 14. Blood 2009;113:5848-56.

37 Tanegashima K, Tsuji K, Suzuki K, et al. Dimeric peptides of the C-terminal region of CXCL14 function as CXCL12 inhibitors. FEBS Lett 2013;587:3770-5.

38 Tanegashima K, Suzuki K, Nakayama Y, et al. CXCL14 is a natural inhibitor of the CXCL12-CXCR4 signaling axis. FEBS Lett 2013;587:1731-5.

39 Meuter S, Schaerli P, Roos RS, et al. Murine CXCL14 is dispensable for dendritic cell function and localization within peripheral tissues. Mol Cell Biol 2007;27:983-92

40 Nara N, Nakayama Y, Okamoto S, et al. Disruption of CXC motif chemokine ligand-14 in mice ameliorates obesity-induced insulin resistance. J Biol Chem 2007:282:30794-803.

41 Tanegashima K, Okamoto S, Nakayama Y, et al. CXCL14 deficiency in mice attenuates obesity and inhibits feeding behavior in a novel environment. PLOS ONE 2010;5:e10321.

42 Li J, Gao J, Yan D, et al. Neutralization of chemokine CXCL14 (BRAK) expression reduces $\mathrm{CCl} 4$ induced liver injury and steatosis in mice. Eur J Pharmacol 2011:671:120-7.

43 De Minicis S, Seki E, Uchinami H, et al. Gene expression profiles during hepatic stellate cell activation in culture and in vivo. Gastroenterology 2007;132: 1937-46.

44 Burke ML, McManus DP, Ramm GA, et al. Co-ordinated gene expression in the live and spleen during Schistosoma japonicum infection regulates cell migration. PLOS Negl Trop Dis 2010;4:e686

45 Michelotti GA, Xie G, Swiderska M, et al. Smoothened is a master regulator of adult liver repair. J Clin Invest 2013;123:2380-94.

46 Augsten $\mathrm{M}$, Hägglöf $\mathrm{C}$, Olsson $\mathrm{E}$, et al. CXCL14 is an autocrine growth factor for fibroblasts and acts as a multi-modal stimulator of prostate tumor growth. Proc Natl Acad Sci USA 2009;106:3414-19.

47 Schwarze SR, Luo J, Isaacs WB, et al. Modulation of CXCL14 (BRAK) expression in prostate cancer. Prostate 2005;64:67-74.

48 Yu M, Gipp J, Yoon JW, et al. Sonic hedgehog-responsive genes in the fetal prostate. J Biol Chem 2009;284:5620-9.

49 Shaykhiev R, Sackrowitz R, Fukui T, et al. Smoking-induced CXCL14 expression in the human airway epithelium links chronic obstructive pulmonary disease to lung cancer. Am J Respir Cell Mol Biol 2013:49:418-25.

50 Wente MN, Mayer C, Gaida MM, et al. CXCL14 expression and potential function in pancreatic cancer. Cancer Lett 2008:259:209-17.

51 Lauth M, Toftgård R. Hedgehog signaling and pancreatic tumor development. $A d v$ Cancer Res 2011;110:1-17.

52 Choe C, Shin YS, Kim SH, et al. Tumor-stromal interactions with direct cell contacts enhance motility of non-small cell lung cancer cells through the hedgehog signaling pathway. Anticancer Res 2013;33:3715-23.

53 Gache Y, Brellier F, Rouanet S, et al. Basal cell carcinoma in Gorlin's patients: a matter of fibroblasts-led protumoral microenvironment? PLOS ONE 2015;10: e0145369

54 Ermilov AN, Kumari A, Li L, et al. Maintenance of taste organs is strictly dependent on epithelial hedgehog/GLI signaling. PLoS Genet 2016;12:e1006442. 